\title{
PDR status for TROPOMI, the Sentinel 5 Precursor instrument for air quality and climate observations
}

\author{
Johan de Vries*a, Robert Voors ${ }^{\mathrm{a}}$, Gerard Otter ${ }^{\mathrm{b}}$, Nick van der Valk ${ }^{\mathrm{b}}$, Ilse Aben ${ }^{\mathrm{c}}$, Ruud Hoogeveen ${ }^{\mathrm{c}}$, \\ Ralph Snel ${ }^{\mathrm{c}}$, Quintus Kleipool ${ }^{\mathrm{d}}$, Pepijn Veefkind ${ }^{\mathrm{d}}$ \\ ${ }^{a}$ Dutch Space B.V. Mendelweg 30 - 2333 CS Leiden, The Netherlands \\ ${ }^{\mathrm{b}}$ TNO Netherlands Organization for Applied Technological Research, Stieltjesweg 1, $2628 \mathrm{CK}$ \\ Delft \\ 'SRON Netherlands Institute for Space Research, Sorbonnelaan 23584 CA Utrecht, The \\ Netherlands \\ dKNMI Royal Netherlands Meteorological Institute, Wilhelminalaan 10, 3732 GK De Bilt
}

\begin{abstract}
The Tropospheric Monitoring Instrument (TROPOMI) is being developed for launch in 2014 on GMES Sentinel 5 Precursor satellite. Global Monitoring for Environment and Security (GMES) is a joint initiative of the European Community (EC) and of the European Space Agency (ESA). TROPOMI is a passive sun backscatter spectrograph using the ultraviolet-to-SWIR wavelengths to have good sensitivity down to the surface. Together with its spatial resolution of $7 \times 7 \mathrm{~km}^{2}$ it allows good observations of sources and sinks of air quality and climate related gases and aerosols. This spatial resolution results in a high fraction of cloud-free observations and is combined with a wide cross-flight swath to allow daily coverage of the complete Earth.

The instrument has as predecessors the OMI instrument (Ozone Monitoring Instrument) on NASAs AURA satellite and the SCIAMACHY instrument on ESAs ENVISAT, where the push broom concept is derived from OMI and the SWIR observations from SCIAMACHY.

The wavelength bands of TROPOMI are a UV band $(270-320 \mathrm{~nm})$ for the ozone profile and $\mathrm{SO}_{2}$ products, a UVIS band ( $320-500 \mathrm{~nm}$ ) for $\mathrm{O}_{3}, \mathrm{NO}_{2}, \mathrm{BrO}, \mathrm{HCHO}$ and OClO total columns, a NIR band (675 - $775 \mathrm{~nm}$ ) for clouds and $\mathrm{H}_{2} \mathrm{O}$ columns and a SWIR band $(2305-2385 \mathrm{~nm})$ for $\mathrm{CH}_{4}$ and $\mathrm{CO}$ columns and the $\mathrm{HDO} / \mathrm{H}_{2} \mathrm{O}$ ratio.

The paper will give an overview of the challenges and current performances.

The TROPOMI UVN module is funded by the Netherlands and the SWIR module and platform is provided via ESA. The instrument development is now a truly European programme with contributions from several countries.
\end{abstract}

Keywords: atmospheric remote sensing, UV-SWIR spectrograph, hyperspectral imaging

\section{INTRODUCTION}

The Tropospheric Monitoring Instrument (TROPOMI) is being developed for launch in 2014 on GMES Sentinel 5 Precursor satellite. Global Monitoring for Environment and Security (GMES) is a joint initiative of the European Community (EC) and of the European Space Agency (ESA). The objectives are to measure the trace gases and aerosols that impact air quality and climate and to better constrain the sources and improve understanding of the trace gas related chemical processes.

TROPOMI is a passive sun backscatter spectrograph using the ultraviolet-to-SWIR wavelengths. These wavelengths have a good sensitivity down to the surface and in combination with the spatial resolution of $7 \times 7 \mathrm{~km}^{2}$ allows for unprecedented observations of sources and sinks of air quality and climate related gases and aerosols. The spatial resolution results in a high fraction of cloud-free observations ${ }^{1}$ and is combined with a wide cross-flight swath $\left(104^{\circ}\right)$ to allow daily coverage of the complete Earth.

Sensors, Systems, and Next-Generation Satellites XV, edited by Roland Meynart, Steven P. Neeck, Haruhisa Shimoda, Proc. of SPIE Vol. 8176, 817609 - @ 2011 SPIE · CCC code: 0277-786X/11/\$18 · doi: 10.1117/12.898027 
The ultraviolet-to-SWIR wavelength range allows TROPOMI to measure key atmospheric constituents such as ozone $\left(\mathrm{O}_{3}\right)$, nitrogen dioxide $\left(\mathrm{NO}_{2}\right)$, carbon monoxide $(\mathrm{CO})$, sulphur dioxide $\left(\mathrm{SO}_{2}\right)$, methane $\left(\mathrm{CH}_{4}\right)$, formaldehyde $\left(\mathrm{CH}_{2} \mathrm{O}\right)$, aerosols and clouds.

The instrument has as most direct predecessors OMI (Ozone Monitoring Instrument) on NASAs AURA satellite ${ }^{2,3}$ and SCIAMACHY on ESAs ENVISAT ${ }^{4}$, where the push broom concept is derived from OMI and the SWIR observations from SCIAMACHY.

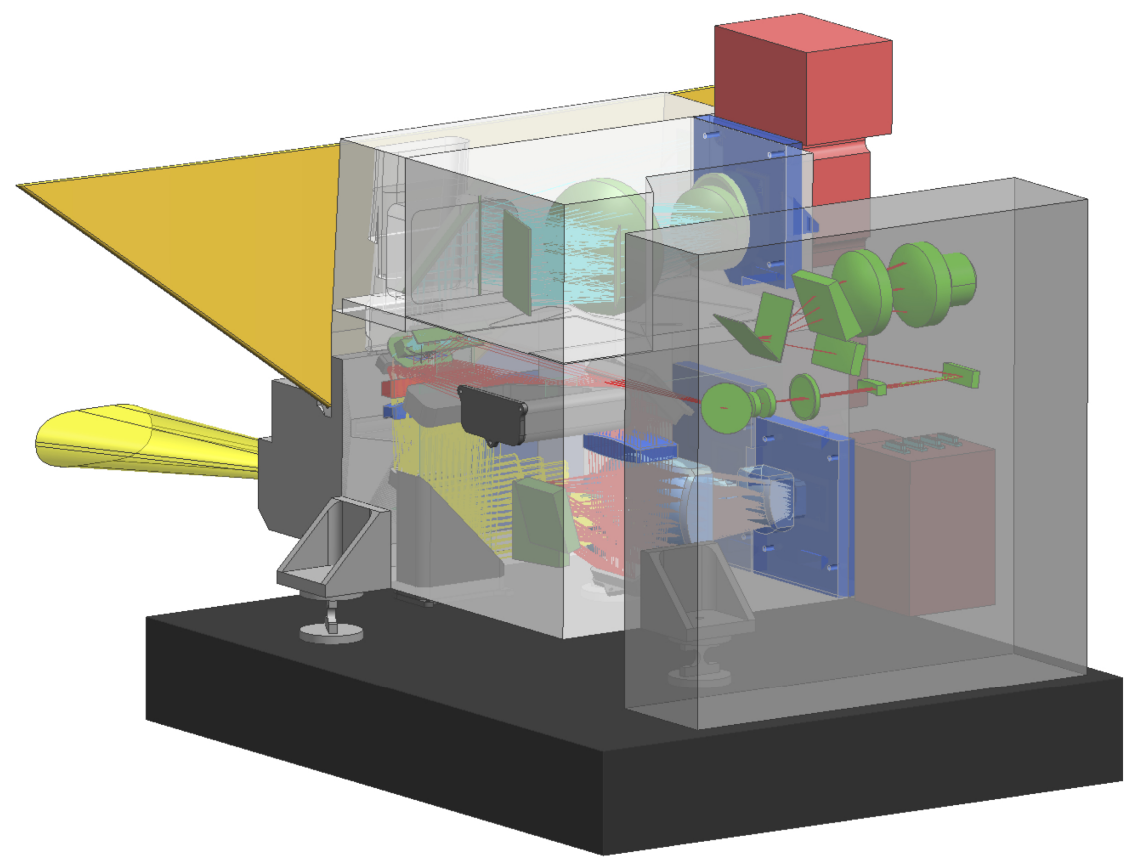

Figure 1 TROPOMI instrument layout with the SWIR module in front and the UVN in the centre; the telescope is at the rear with the $108^{\circ}$ wide angle field-of-view

The instrument layout is shown in Figure 1. It has a UVN module with UV, UVIS and NIR bands and a cooled SWIR module with a single SWIR band. The figure shows how the UVN module and telescope and the SWIR module are mounted on a common base plate. The telescope and UVN module have a common structure and the light from the telescope is fed into the SWIR module via relay optics.

Each of the bands has a separate detector as shown in Table 1 together with their spectral and spatial properties and signal-to-noises. The spatial sampling is mostly $7 \times 7 \mathrm{~km}^{2}$ with the exception of bands 1 and 6 . Band 1 has a larger spatial sampling to allow good signal-to-noises given the low radiances for these wavelengths. Band 6 is used to obtain the most important cloud products. It is read at higher spatial resolution to have as good as possible co-registration of these cloud products and the other bands (see section 5). The signal-to-noises mentioned in Table 1 for this band are valid after on-ground increase of the ground pixels to the original $7 \times 7 \mathrm{~km}^{2}$.

There is a similar situation for bands 1 and 2 where the spectral sampling is increased: also for these bands the signalto-noises are valid for a factor 3 oversampling of the spectral resolution mentioned. 
Table 1 TROPOMI spectral bands

\begin{tabular}{|c|c|c|c|c|c|c|c|}
\hline Detector & \multicolumn{2}{|c|}{ UV } & \multicolumn{2}{|c|}{ UVIS } & \multicolumn{2}{|c|}{ NIR } & SWIR \\
\hline Band & 1 & 2 & 3 & 4 & 5 & 6 & 7 \\
\hline $\begin{array}{l}\text { Spectral } \\
\text { range }[\mathrm{nm}]\end{array}$ & $270-300$ & $300-320$ & $310-405$ & $405-500$ & $675-725$ & $725-775$ & $2305-2385$ \\
\hline $\begin{array}{l}\text { Spectral } \\
\text { resolution } \\
{[\mathrm{nm}]}\end{array}$ & 1.0 & 0.5 & 0.55 & 0.55 & 0.5 & 0.5 & 0.25 \\
\hline $\begin{array}{l}\text { Spectral } \\
\text { sampling } \\
{[\mathrm{nm}]}\end{array}$ & 0.065 & 0.065 & 0.2 & 0.2 & 0.1 & 0.1 & $<0.1$ \\
\hline $\begin{array}{l}\text { Spatial } \\
\text { sampling } \\
{\left[\mathrm{km}^{2}\right]}\end{array}$ & $21 \times 28$ & $7 \times 7$ & $7 \times 7$ & $7 \times 7$ & $7 \times 7$ & $2.3 \times 1.8$ & $7 \times 7$ \\
\hline $\begin{array}{l}\text { Minimum } \\
\text { signal-to- } \\
\text { noise }\end{array}$ & $\left.100-800^{1}\right)$ & $\left.90-700^{1}\right)$ & $\left.800-1000^{1}\right)$ & $\left.800-1000^{1}\right)$ & $\left.100-500^{1}\right)$ & $\left.100-500^{1}\right)$ & $\left.100-120^{2}\right)$ \\
\hline
\end{tabular}

${ }^{1}$ ) The minimum signal to noise is specified for a scene with an albedo of $2 \%$ and the Sun in zenith.

${ }^{2}$ ) The minimum signal to noise is specified for a scene with an albedo of $5 \%$ and solar zenith angle of $70^{\circ}$

The UVIS and NIR bands make use of the same spectrograph slit whereas the UV and SWIR have separate slits. This allows having a wider slit for the UV to compensate for the lower radiance for these wavelengths and for the SWIR it allows to have the slit included in the cooled SWIR module. The different slits result in slightly different viewing angles in the flight direction.

The overall mass of the instrument is slightly over $200 \mathrm{~kg}$. This is fairly low for the instruments capability and this is possible because the SWIR band uses a specially developed Silicon immersed grating ${ }^{5}$ (see Figure 2).

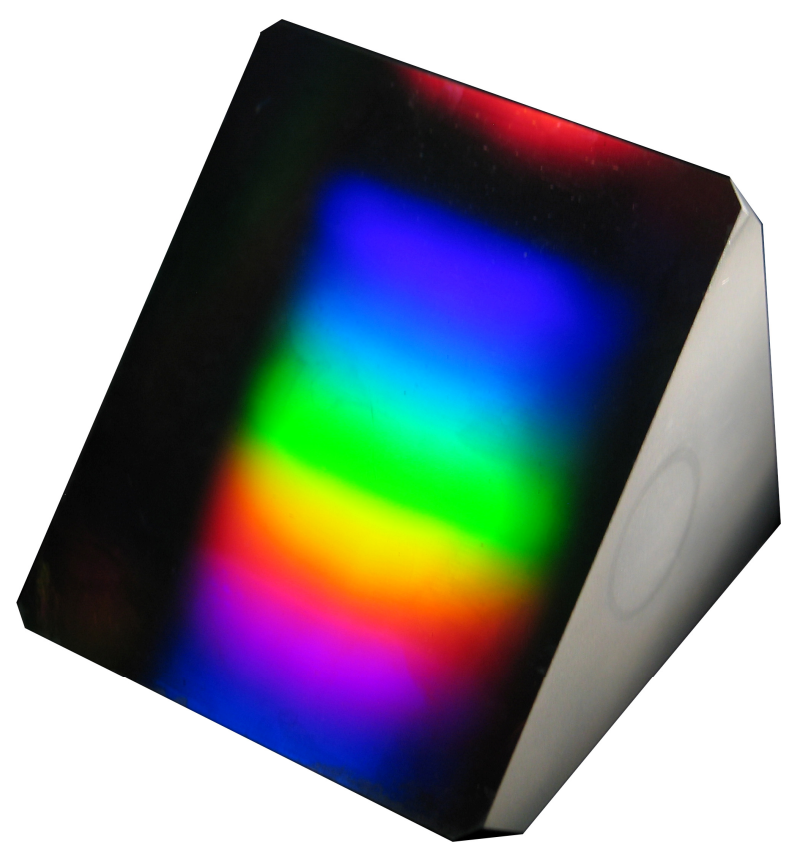

Figure 2 Photograph of the immersed grating developed for the TROPOMI SWIR band ${ }^{5}$; using this grating as compared to a conventional grating allowed a considerable reduction of the size of the SWIR band. The development was done by SRON and TNO. 


\section{PROGRAM STATUS}

The TROPOMI project is led by Dutch Space on the industrial side, by KNMI and SRON for science and by NSO and ESA for programmatic aspects.

The instrument development passed its Instrument-PDR review (IPDR) in May 2011. The IPDR was conducted as a top down review and sub-system PDRs follow in the remainder of this year. This means that a lot of detail information is presently becoming available. Because this information could not be included in the IPDR, the sub-system PDRs will be followed by an IPDR wrap-up meeting in December 2011.

The relevant sub-systems are the UVN module, funded by the Netherlands Space Office (NSO), the SWIR module, the Instrument Control Unit (ICU), the Telescope Support Structure (TSS), the Radiant Cooler (RC) and the ground support equipment (GSE), funded by ESA. The UVN module contains the UVN spectrograph bands, the telescope and calibration unit. This unit will not have a separate PDR because the unit is developed by the prime-team of Dutch Space and TNO. The SWIR module is the cooled module containing the SWIR spectrograph and is developed by SSTL in the UK. The SWIR detector will be from Sofradir (F) and is controlled by Front-End Electronics developed by SRON. The SWIR module will have its PDR in October. The ICU is the main electronics unit including clock sequencers for detector readout and is developed by RUAG in Sweden; the TSS (Astrium Germany) is the structure carrying the UVN with telescope and the SWIR modules. Together with the RC and GSE the PDRs for these units will be in fall 2011.

As part of the UVN module we have the UVN detector modules (UVN-DEM) with a PDR in October and as part of the UVN-DEM the UVN CCDs as developed by E2V in the UK. The UVN CCDs had a Test Readiness Review (TRR) in September and have actual first hardware test results in development model detectors.

With the many parties involved, TROPOMI is a large and challenging instrument project.

\section{DETECTOR DEVELOPMENT STATUS}

The UVN detector developed for TROPOMI is a back illuminated $1 \mathrm{~K} \times 1 \mathrm{~K}$ pixel frame transfer CCD with a pixel pitch of $26 \mu \mathrm{m}$. The device is developed by E2V in the UK and has different coatings for the different wavelength bands to allow maximizing the quantum efficiency and minimizing interference structures for the NIR.

The device is operated in non-inverted mode (NIMO). Despite the higher intrinsic dark current (surface dark current is not suppressed) it has a number of advantages. First, the so-called ellipsoid effect present in inverted mode (IMO) is not present. It can also be avoided by keeping the pixel filling below $50 \%$ of the nominal full well but in view of the already high clock frequency of $5 \mathrm{MHz}$ this is deemed unacceptable. A further advantage of NIMO is the much lower power consumption This in turn means that with the same power dissipation, a much lower temperature can be obtained, thereby repairing much of the increase in dark current. Since the largest contribution to the dark current will come from the surface of the CCD, the contribution from random telegraph signal (RTS) will be much lower. In addition the lower temperature will not only decrease the bulk dark current but also the RTS, both in amplitude and in time scale. At the proposed operating temperature the time scale of any RTS will probably be long enough such that any RTS that may be present can be corrected.

The device has metal buttresses to have the line transfer time as low as possible to minimize exposure smear. Having metal buttresses means that with current days technologies we are bound to 2 phase parallel clocking. This results in a lower pixel full well as compared to 4 phase clocking but was accepted to have a low development risk.

First test results with a front illuminated breadboard detectors are now available. They show most performances are as expected. There is no sign of RTS pixels in the test devices.

For the SWIR, TROPOMI uses the off-the-shelve Sofradir SATURN detector. This is a HgCdTe-based CMOS detector with $1000 \times 256$ pixels of $30 \mu \mathrm{m}$ pitch. The detector is, apart from the number of pixels, similar to the MARS detector which was used successfully in a SWIR spectrograph breadboard ${ }^{6}$. 


\section{OPERATIONAL FLEXIBILITY}

TROPOMI is a very flexible instrument in terms of the readout of its detectors. The most important instrument settings are as follows.

- To avoid saturation in the detectors, there are up to 25 detector readouts during the spatial sampling satellite travel distance. TROPOMI allows users to set the exposure times with step size $1 \mathrm{~ms}$ for the UVN and $200 \mu \mathrm{s}$ for the SWIR and the number of exposures to be co-added into the spatial sampling distance in the flight direction.

- The UVN module CCDs bin a programmable number of pixels to have the wanted sampling in the swath direction, nominally 4 detector pixels are binned to have $7 \mathrm{~km}$ at nadir. Because sampling measured on ground increases with swath angle, it is possible to have lower binning factors towards the extreme swath angles.

- The UVN module CCDs allow binning groups of pixel rows below and above the illuminated regions to have stray light estimates and also to bin covered rows on top and the bottom of the CCDs for exposure smear and dark current. Gains for these rows are selected separately to allow fair signal-to-noises.

- For each UVN band, it is possible to select the CCD output amplifier gain and the ADC gain, separately for each row.

The exposure time settings are to be used to optimize signal-to-noises for different latitudes and for special cases such as ozone hole conditions. Since the exposure times for all bands have to fit into the same satellite travel time, it is also possible to adjust the travel time and thereby the spatial sampling in the flight direction.

The flexibility in selecting the exposure times introduces a risk of EMC effects in the detector readout. This risk is minimized by including ADCs in the detector proximity electronics and thereby having digital signals in the harness between detector modules and electronics unit. On the other hand, the detectors are read at a frequency of $5 \mathrm{MHz}$ which is high enough to be cautious.

This risk is mitigated by synchronizing the different detectors. This is achieved by implementation of a few simple rules.

- During a frame transfer of any UVN detector, there shall be no readout of any other detector

- During a line transfer (of the storage section into the register) of any UVN detector, there shall be no readout of any other detector.

- Frame transfers, line transfers and readout shall not be interrupted.

It is possible to switch it off in case the EMC risk does not show up in later hardware phases

\section{CO-REGISTRATION}

Co-registration means that all wavelengths of a given detector row view in the same direction, both in the across-flight and in the flight direction. Co-registration is important because level 1-2 product retrieval algorithms assume all wavelengths in the Level 1 product observe the same air mass. There are a number of hardware effects that impact the co-registration performance.

In the flight direction, the different slits for UV, UVIS/NIR and SWIR lead to a swath dependent offset as shown in Figure 3. The effect for the UV is similar but this is not so critical as this band observes the upper atmosphere with few clouds and scene variation. 


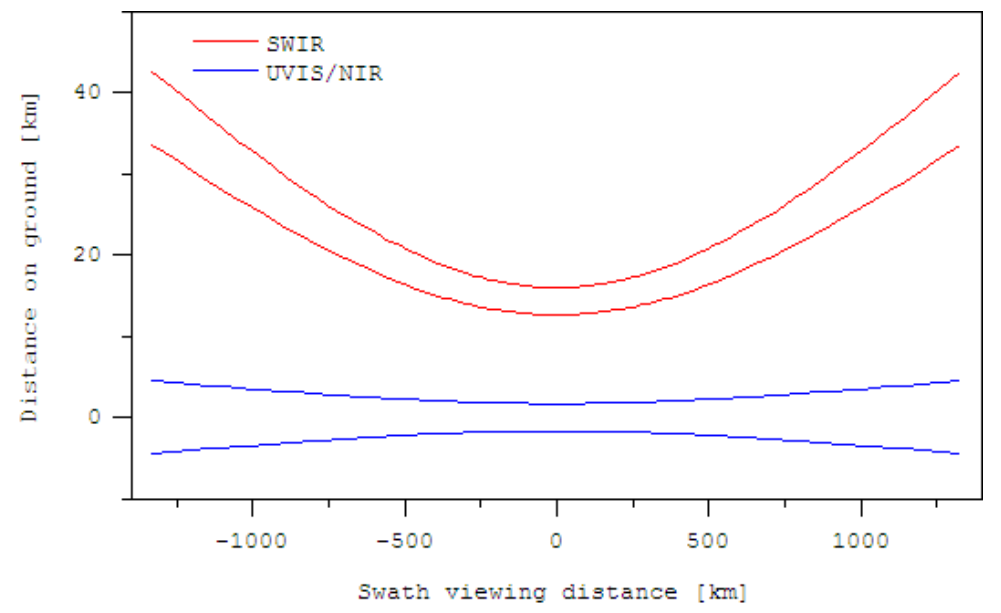

Figure 3 UVIS/NIR and SWIR slits projected onto the Earth surface, showing the displacement, increase with swath angle and relative curvature in the flight direction; the two bottom curves mark the begin and end of the slit projection for the UVIS/NIR and the two upper curves for the SWIR

In the swath direction and within detector bands, there are the cushion-shaped distortions related to using gratings. These are minimized as much as possible in the optical design but there will be remnants due to manufacturing tolerances.

In the swath direction the most difficult effects are between the detector bands, as these require sub-detector pixel accuracies in detector mounting and optical element alignment and the accuracies include orbital effects due to the changing thermal environment.

The most critical co-registration performance is between the NIR and the SWIR and the NIR and the UVIS bands because the NIR band yields the cloud product needed to obtain accurate air mass estimates for the trace gas products from the SWIR and UVIS.

Therefore we downlink the NIR data at improved spatial sampling and interpolate the NIR data towards the SWIR and the UVIS viewing. In the swath direction, this is possible by reducing the detector pixel binning from 4 to 2 and thereby have a spatial sampling of $3.5 \mathrm{~km}$. In the flight direction, the co-addition time is reduced by a factor 3 and thereby the spatial sampling is about $2.3 \mathrm{~km}$. Given the relevant resolutions, this is sufficient for interpolation. Interpolation is seen as an effective correction of the inter-channel co-registration errors. The accuracy of the knowledge of the pointing difference between channels and its stability form now the most important remaining error. The stability of the coregistration during flight is estimated to be within $10 \%$ of a ground pixel which is sufficiently small.

\section{HETEROGENEOUS SCENES}

Trace gas products from instruments such as TROPOMI are normally derived from reflectance spectra, the ratio of Earth radiance and sun irradiance measurements. The absorption signatures in these spectra can be small, e.g. in the order of a percent in the case of minor absorbing gases. Therefore, if we want an accuracy in the product of a few percent, we need the reflectance spectra to be free from any distortion on the $10^{-3}$ to $10^{-4}$ level and with a very accurate wavelength definition in the order of 1/100 of a spectral sampling distance.

There are several mechanisms introducing such distortions or features:

- Sun measurements use a diffuser to convert irradiance into radiance entering the telescope; because of the good spectral and spatial resolution coherence effects will show up as seemingly random spikes or features; the Earth spectra do not use a diffuser and therefore the features are present in the reflectance spectra

- The polarization scrambler consists of a stack of four birefringent wedges and result in wavelength and viewing angle dependent modulations of the signals; these modulations vary in the Earth measurements with polarization but they are constant in the sun measurements as this is not polarized 
- Varying pixel-to-pixel variation in the detector sensitivity (PRNU) in combination with a varying scene and pixel binning leads to small errors in Earth radiance measurements; sun measurements do not have the effect because the sun is spatially constant via the diffuser

- Non-uniform illumination of the slit in the across slit direction leads to distortion of the slit function and effectively in a wavelength shift and radiometric effect

The latter is the heterogeneous scenes effect and is the topic of this section.

Figure 4 shows the basics of an OMI-type spectrograph, showing the transfer of flight direction spatial information towards slit illumination and from there onto the spectral direction of the 2D detector.

The left graph shows light beams from different directions entering the telescope and being imaged on different edges of the slit. The right graph shows a spectrograph where the beams are projected on different locations of the detector. If the scene is constant, this leads to the desired slit function (or SRF, the Spectral Radiance Function) imaged onto the detector. If the illumination of the slit is not uniform, the beams have different radiance content and cause a distortion of the slit function. This distortion causes a radiometric error, and, because the barycentre of the slit function is changed, to a wavelength error.

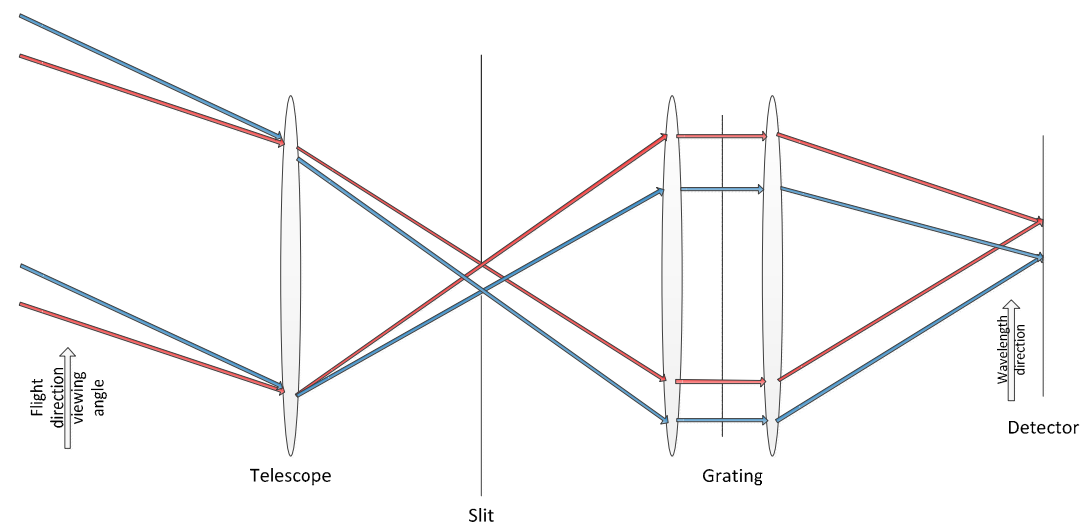

Figure 4 Basic push-broom spectrograph showing how flight direction spatial information on the left is transferred to the spectral response function on the right; colour separation from the grating is left out of the graph

Below we show results from model calculations on the heterogeneous scenes effect. Figure 5 shows in the left graph an input scene which varies in the flight direction, but excluding the motion of the satellite. In the right graph we see the corresponding signal on the detector.
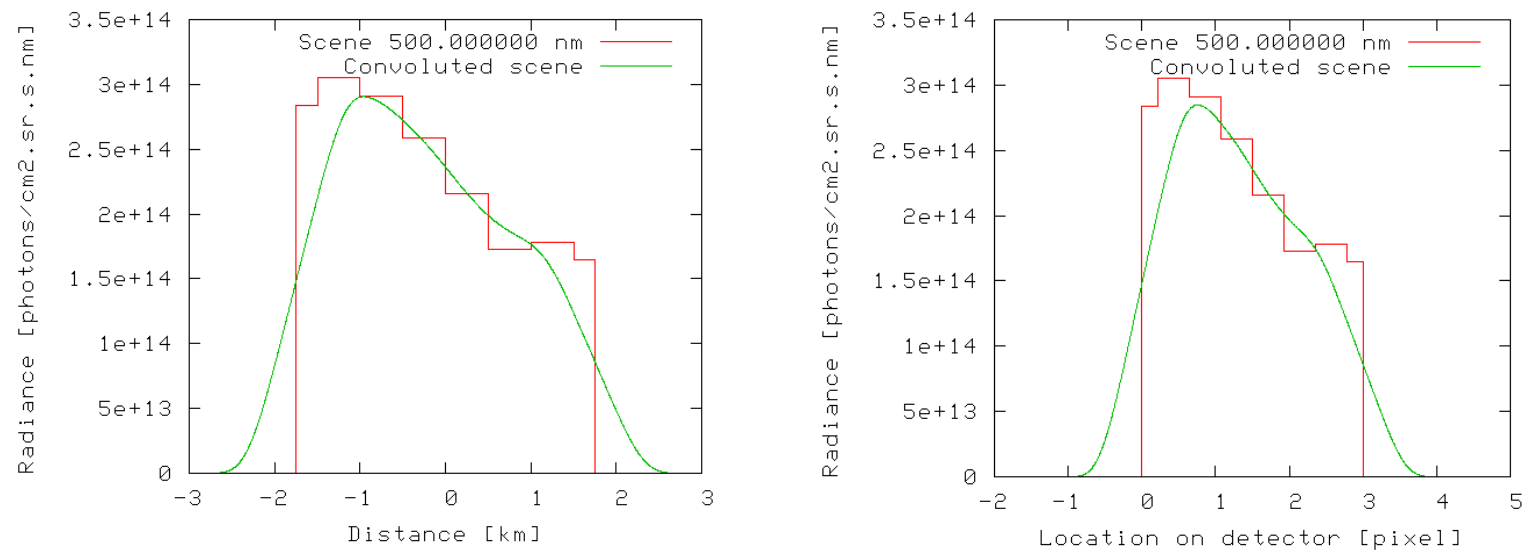

Figure 5 Staring observation of a heterogeneous scene for the UVIS band at $500 \mathrm{~nm}$; the scene is the $500 \mathrm{~m}$ resolution histogram-type curve and the smooth curve is what the instrument actually observes via convolving with an optics and detector point-spread function; the left graph shows the spatial effect and the right graph the spectral effect 
Including the motion of the satellite, Figure 6 shows on the left side resulting slit functions or SRF (Spectral Radiance Function) for the 24 UVIS exposures in a co-addition time of $1 \mathrm{~s}$. Some of the slit functions are clearly sloped due to the slope from Figure 5, other exposures are in a constant part of the scenes and the corresponding slit functions have a flat top. The right graph in Figure 6 shows the radiance convolved with the slit functions for each of the exposures.
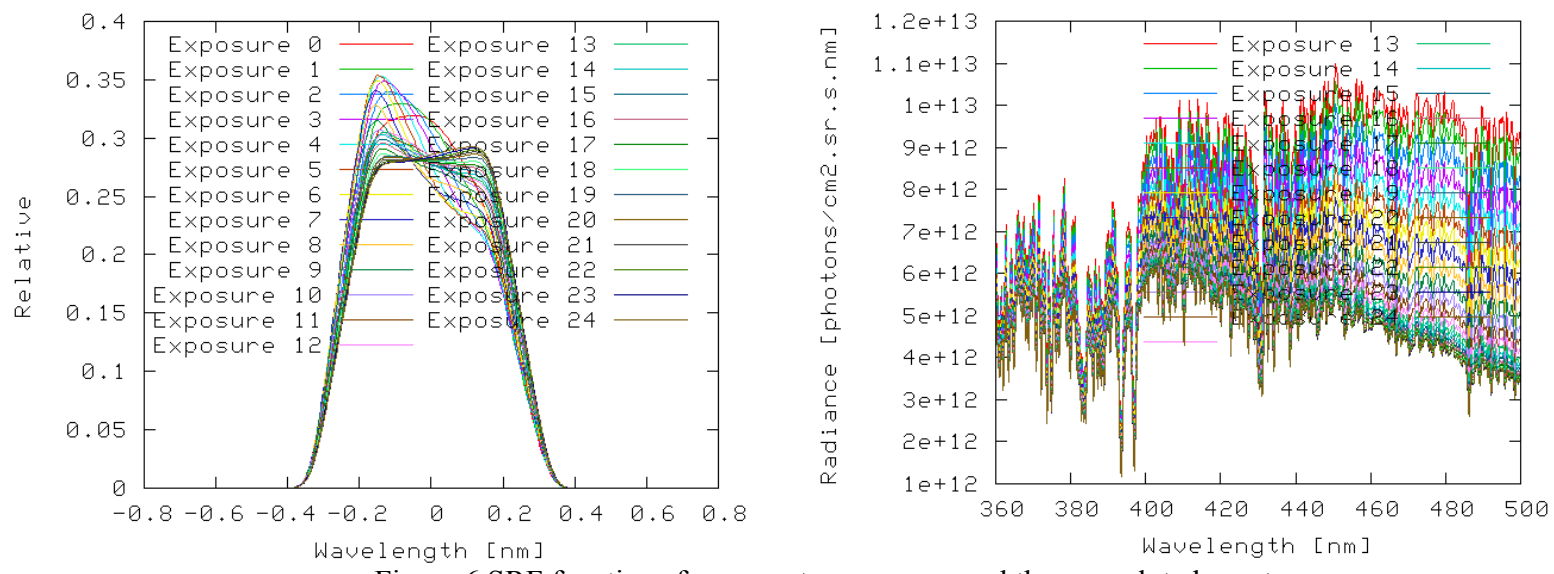

Figure $6 \mathrm{SRF}$ functions for separate exposures and the convoluted spectra

After co-adding the exposures, we obtain the signal as produced by the instrument. Comparing this with a signal from an averaged constant radiance spectrum, it is possible to compute the relative error as a function of wavelength, as follows.

$$
a(\lambda)=\frac{L(\lambda)-L_{\operatorname{rgf}}(\lambda)}{L(\lambda)+L_{\operatorname{rat}}(\lambda)}
$$

The result is shown in Figure 7. In Figure 8 we show results from a similar model calculation for the NIR band. The scene change in this case is about $15 \%$, so less demanding as compared to the UVIS scene change.

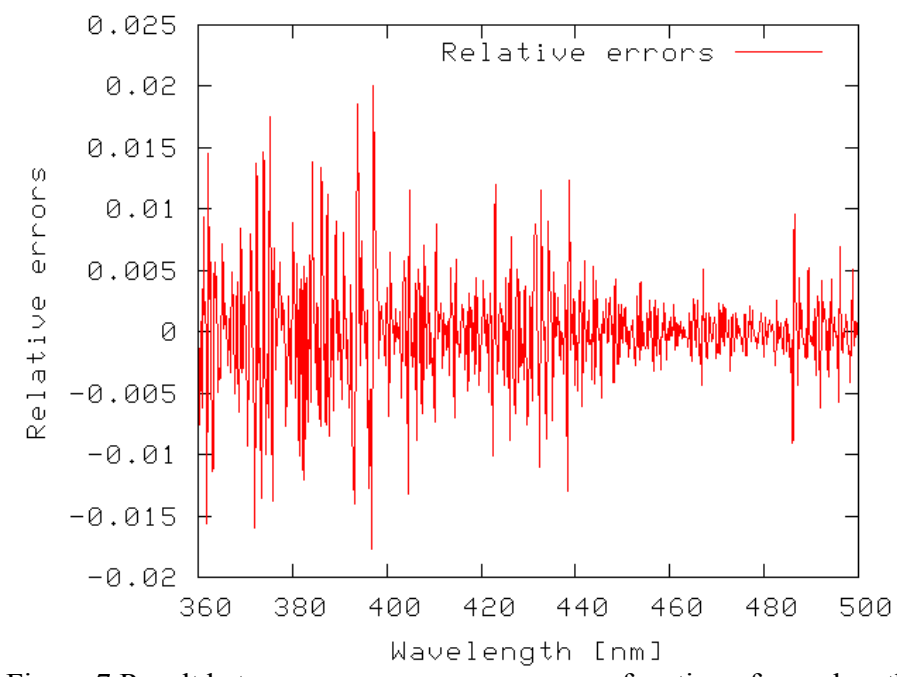

Figure 7 Result heterogeneous scenes errors as a function of wavelength 

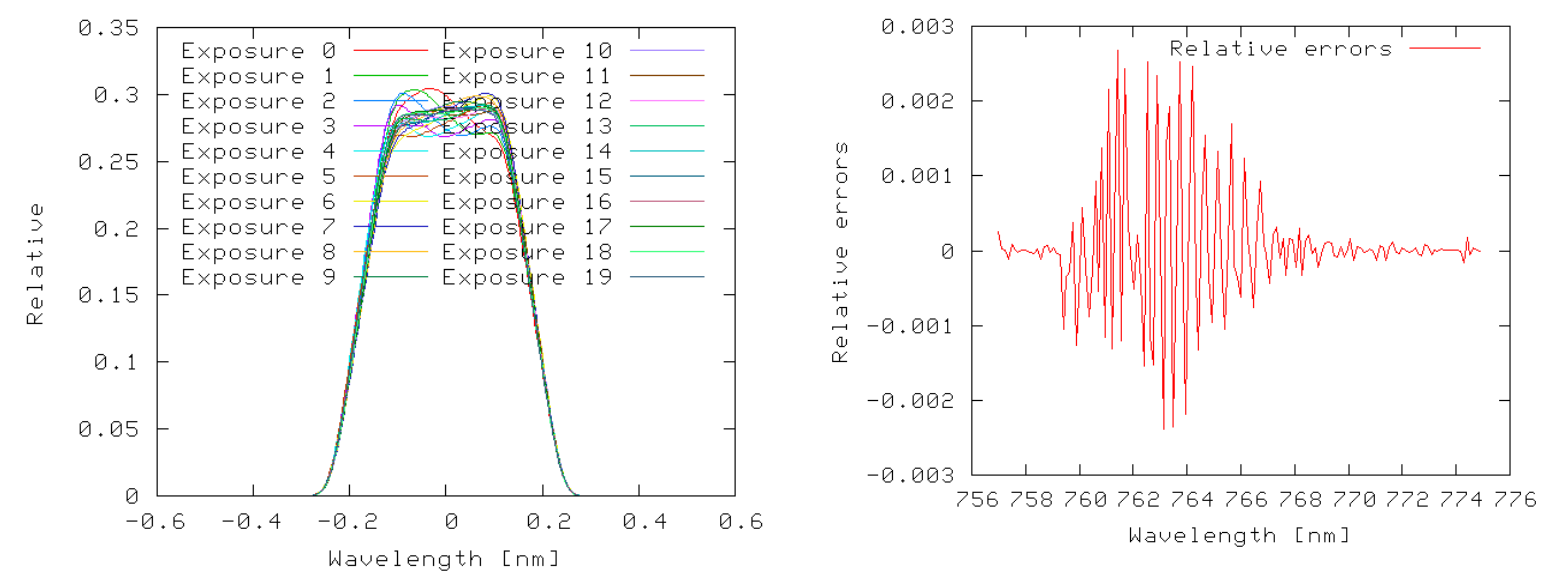

Figure 8 Result heterogeneous scenes errors as a function of wavelength

The objective of these simulations is to allow developing a correction algorithm to be included in the Level 0-1b processor. The OMI instrument has for this purpose a wavelength correction algorithm. This algorithm adjusts the wavelength definition of the spectra to for a major part correct for the errors seen in Figures 7 and 8. For TROPOMI and the Sentinel 5 UVNS, the TROPOMI follow-up, we intend to further improve the algorithm.

\section{CONCLUSION}

The paper has described in some level of detail the TROPOMI instrument to be launched on ESAs Sentinel 5 Precursor satellite in 2014. The topics covered are at this moment relevant for the instrument development.

As compared to its predecessors, the SCIAMACHY on ESAs ENVISAT and the OMI on NASAs AURA satellite, TROPOMI is a major step forward in terms of spatial resolution and accuracy. It is challenging in terms of instrument development and exciting in terms of the expected science output. We look forward to Development Model tests in fall 2012 and Flight Model tests one year later.

\section{REFERENCES}

[1] Krijger, J. M., Van Weele, M., Aben, I., \& Frey, R. (2007). Technical Note: The effect of sensor resolution on the number of cloud-free observations from space. Atmos. Chem. Phys., 7(11), 2881-2891.

[2] Levelt, P. F., van den Oord, G. H. J., Dobber, M. R., Malkki, A., Visser, H., de Vries, J., Stammes, P., et al. (2006). The ozone monitoring instrument. IEEE Trans. on Geosc. and Rem. Sens., 44(5), 1093-1101.

[3] Dobber, M. R., Dirksen, R. J., Levelt, P. F., Van Den Oord, G. H. J., Voors, R. H. M., Kleipool, Q., Jaross, G., et al. (2006). Ozone monitoring instrument calibration. Geoscience and Remote Sensing, IEEE Transactions on, 44(5), 12091238 .

[4] Bovensmann, H., Burrows, J. P, Buchwitz, M., Frerick, J., Noël, S., Rozanov, V. V., Chance, K. V., et al. (1999). SCIAMACHY: Mission Objectives and Measurement Modes. Journal of the Atmospheric Sciences, 56(2), 127-150.

[5] Amerongen, A. H. van, Visser, H., Vink, R. J. P., Coppens, T., \& Hoogeveen, R. W. M. (2010). Development of immersed diffraction grating for the TROPOMI-SWIR spectrometer. In R. Meynart, S. P. Neeck, \& H. Shimoda (Eds.), Sensors, Systems, and Next-Generation Satellites XIV (Vol. 7826, p. 78261D). SPIE. doi:10.1117/12.869018 
[6] Hoogeveen, R.W.M., Jongma, R.T., Tol, P.J.J., Gloudemans, A., Aben, I.E.E., de Vries, J., Visser, H., Boslooper, E., Dobber, M., Levelt, P.F., "Breadboarding activities of the TROPOMI-SWIR module", Proc. SPIE, 6744, 67441T (2007).

This document has been produced with the financial assistance of the European Union. The views expressed herein can in no way be taken to reflect the official opinion of the European Union and/or ESA. 